\title{
Berberine enhances the anti-tumor activity of tamoxifen in drug-sensitive MCF-7 and drug-resistant MCF-7/TAM cells
}

\author{
CHUNJIE WEN, LANXIANG WU, LIJUAN FU, XUE ZHANG and HONGHAO ZHOU \\ Institute of Life Sciences, Chongqing Medical University, Chongqing 400016, P.R. China
}

Received September 6, 2015; Accepted June 14, 2016

DOI: $10.3892 / \mathrm{mmr} .2016 .5490$

\begin{abstract}
Berberine, an isoquinoline alkaloid, has been previously demonstrated to possess anti-breast cancer properties. Tamoxifen is widely used in the prevention and treatment of estrogen receptor-positive breast cancer. Thus, the aim of the present study was to assess whether berberine enhanced the anticancer effect of tamoxifen, and the underlying mechanism involved in this combined effect in tamoxifen-sensitive (MCF-7) and tamoxifen-resistant (MCF-7/TAM) cells using MTS, flow cytometry and western blot assays. The results indicated that berberine demonstrated dose- and time-dependent anti-proliferative activity in MCF-7 and MCF-7/TAM cells. Furthermore, the combination of berberine and tamoxifen induced cell growth inhibition more effectively than tamoxifen alone. The present study also demonstrated that combinational treatment is more effective in inducing G1 phase arrest and activating apoptosis compared tamoxifen alone, which may be due to upregulation of P21 expression and downregulation of the B-cell CLL/lymphoma 2(Bcl-2)/Bcl-2 associated X protein ratio. The results of the present study suggested that berberine may potentially be useful as an adjuvant agent in cancer chemotherapy to enhance the effect of tamoxifen, which will be useful for anti-tumor therapy and further research.
\end{abstract}

\section{Introduction}

Breast cancer is the most common type of cancer and leading cause of cancer-related mortality in females with an estimated 1.7 million cases and 521,900 related fatalities in 2012 (1). Breast cancer is the most common type of cancer and was the leading cause of cancer mortality in women in 2008 , and it is estimated that breast cancer will affect five million women worldwide over the subsequent decade (1). Over $60 \%$ of breast cancers are estrogen receptor (ER)-positive, and their

Correspondence to: Professor Honghao Zhou, Institute of Life Sciences, Chongqing Medical University, 1 Xueyuan Road, Chongqing 400016, P.R. China

E-mail: hhzhou2003@163.com

Key words: berberine, tamoxifen, cell cycle, apoptosis, breast cancer development can be stimulated by estrogen and inhibited by ER antagonists, including tamoxifen.

Tamoxifen, broadly used in ER-positive breast cancer prevention and treatment, was approved as the first line anti-estrogen therapy in 1999 by the US Food and Drug Administration (2). Endocrine therapy with tamoxifen for five years has resulted in a $9.2 \%$ absolute reduction in mortality at 15 years, with a $34 \%$ reduction in the breast cancer mortality rate per year (3). However, the emergence of resistant cancer cells limits its therapeutic effectiveness $(4,5)$. Thus, tamoxifen is typically administered in combination with other drugs, reducing the incidence of the development of drug resistance $(6,7)$.

Previously, researchers have taken an increasing interest in combination therapy by associating anti-cancer herbal medicine with chemotherapeutic agents, and have demonstrated significant successes (8-10). Berberine, an isoquinoline plant alkaloid isolated from Coptidis rhizome, regulates multiple targets and may be a promising natural agent with chemotherapeutic potential based on its effect on the expression of various proteins (11-14). Berberine has previously been used to enhance cancer therapy sensitization and to assist chemotherapy by modulating multiple pathways (15-17). These molecular targets of berberine are also involved in growth maintenance and resistance acquisition in tamoxifen-resistant breast cancer. Thus, a combination of berberine with tamoxifen may be an interesting option in the treatment of patients with ER-positive breast cancer.

The current study evaluated the individual and combined effects of berberine and tamoxifen in breast cancer MCF-7 and tamoxifen-resistant MCF-7/TAM cells. The results of the present study demonstrated that berberine does not abolish the anti-tumor effects of tamoxifen, and it induces cell G1 arrest, apoptosis, and significantly enhances the growth inhibition effect of tamoxifen in these cells. The mechanisms underlying these effects involve the regulation of important cell cycle and apoptosis-associated proteins.

\section{Materials and methods}

Materials. MCF-7 human breast cancer cells were provided by the Institute of Biochemistry and Cell Biology, Chinese Academy of Sciences (Shanghai, China). Berberine (Sigma-Aldrich, St. Louis, MO, USA) and tamoxifen (Sigma-Aldrich) were freshly dissolved in dimethyl sulfoxide 
and methanol, respectively, and stored in the dark at $4^{\circ} \mathrm{C}$. The final concentration of vehicle in the culture did not exceed $0.1 \%$ (v/v). RPMI 1640 medium and fetal bovine serum (FBS) were purchased from Gibco (Thermo Fisher Scientific, Inc., Waltham, MA, USA). The 3-(4,5-dimethylthiazol-2-yl)-5-(3carboxymethoxy phenyl)-2-(4-sulfophenyl)-2H-tetrazolium (MTS) assay reagent was purchased from Promega Corporation (Madison, WI, USA). Anti-P21 (goat-anti-rabbit polyclonal; cat. no. 10355-1-AP), cyclin D1 (goat-anti-mouse monoclonal; cat. no. 60186-1-Ig), B-cell CLL/lymphoma 2 (Bcl-2; goat-anti-rabbit polyclonal; cat. no. 12789-1-AP), Bcl-2 associated X protein (Bax; goat-anti-rabbit polyclonal; cat. no. 23931-1-AP), $\beta$-actin (goat-anti-mouse monoclonal; cat. no. 60008-1-Ig) antibodies were purchased from ProteinTech Group, Inc. (Chicago, IL, USA). Anti-mouse and anti-rabbit horseradish peroxidase (HRP)-conjugated (cat. nos. A0428 and A0423, respectively) secondary antibodies were purchased from Beyotime Institute of Biotechnology (Haimen, China).

Establishment of MCF-7/TAM cells. To establish tamoxifen-resistant MCF-7 cell resistant to tamoxifen, MCF-7 cells were exposed to $100 \mathrm{nM}$ tamoxifen in culture. Following 6 months of continuous expose to tamoxifen, these cells exhibited proliferation in medium plus tamoxifen comparable to that of parental cells without tamoxifen, suggesting the development of resistance to the proliferation-inhibitory properties of tamoxifen.

Cell culture and treatment. MCF-7 and tamoxifen-resistant MCF-7/TAM cells were cultured in RPMI 1640 medium supplemented with penicillin $(100 \mathrm{U} / \mathrm{ml})$, streptomycin $(100 \mu \mathrm{g} / \mathrm{ml})$ and $10 \% \mathrm{FBS}$. To maintain tamoxifen resistance, MCF-7/TAM cells were cultured in a medium containing $100 \mathrm{nM}$ of tamoxifen. All cells were maintained in humidified $37^{\circ} \mathrm{C}$ incubators with $5 \% \mathrm{CO}_{2}$.

MTS assay of cell proliferation. The effects of berberine, tamoxifen and their combination on MCF-7 and MCF-7/TAM cell viability were determined by MTS assay. The cells were seeded in 96-well plates at a density of $1 \times 10^{4}$ cells/well and were cultured for $24 \mathrm{~h}$. Cells were treated with increasing doses of berberine $(0,20,40,80,120$ and $160 \mu \mathrm{M})$ for 24 , 48 and $72 \mathrm{~h}$, respectively. MCF-7 cells were exposed to different concentrations of tamoxifen $(0.5,1,2,4$ and $8 \mu \mathrm{M})$ with or without $20 \mu \mathrm{M}$ berberine for $48 \mathrm{~h}$ and MCF-7/TAM cells were exposed to different concentrations of tamoxifen $(1,2,4,8$ and $16 \mu \mathrm{M})$ with or without $20 \mu \mathrm{M}$ berberine for 48 h. Subsequently, MCF-7 and MCF-7/TAM cells were then exposed to different concentrations of tamoxifen with or without $20 \mu \mathrm{M}$ berberine for $48 \mathrm{~h}$. Subsequently, $20 \mu \mathrm{l}$ MTS was added to each well and incubated for $4 \mathrm{~h}$ at $37^{\circ} \mathrm{C}$. The absorbance was examined at $490 \mathrm{~nm}$ using an enzyme-linked immunosorbent detector (BioTek Synergy2; BioTek Instruments, Inc., Winooski, VT, USA).

Flow cytometry analysis of cell cycle distribution. Cells were plated in 6-well plates and cultured for $24 \mathrm{~h}$. Then MCF-7 and MCF-7/TAM cells were treated with tamoxifen $(1 \mu \mathrm{M})$, berberine $(20 \mu \mathrm{M})$, their combination $(20 \mu \mathrm{M}$ berberine and
$1 \mu \mathrm{M}$ tamoxifen) or vehicle. Following treatment for $48 \mathrm{~h}$, cells were collected, washed twice with phosphate-buffered saline (PBS), fixed with ice cold $70 \%$ ethanol at $-20^{\circ} \mathrm{C}$ overnight, centrifuged and resuspended in $300 \mu \mathrm{l}$ PBS containing $0.5 \mathrm{mg} / \mathrm{ml}$ propidium iodide (PI; eBioscience, San Diego, CA, USA) and $0.1 \mathrm{mg} / \mathrm{ml}$ of Rnase A (eBioscience, San Diego, CA, USA). Subsequent to incubation for $30 \mathrm{~min}$ at $37^{\circ} \mathrm{C}$, samples were evaluated using the Cytomics FC 500 flow cytometer (Beckman Coulter, Inc., Brea, CA, USA).

Flow cytometry analysis of apoptosis. Briefly, following the described treatment with berberine and tamoxifen, floating and adherent cells (detached using trypsin) were collected, washed twice in cold PBS, and then suspended in binding buffer (BB (BB, BD Pharmingen, San Diego, CA, USA). Cells (1x10 ${ }^{5}$ in $100 \mu \mathrm{lBB})$ were then stained using $2.5 \mu \mathrm{l}$ Annexin V-fluorescein isothiocyanate (FITC; BD Pharmingen) and $20 \mu 125 \mathrm{X}$ PI solution. Cells were incubated in the dark at room temperature for $15 \mathrm{~min}$, diluted using additional $400 \mu \mathrm{lBB}$ and analyzed with Cytomics FC 500 flow cytometer (Beckman Coulter, Inc.).

Western blot analysis. Cells were treated as described, then placed on ice and washed twice with cold PBS. Cells were then scraped into a radioimmunoprecipitation lysis buffer (Beyotime Institute of Biotechnology) supplemented with proteinase inhibitor phenyl methyl sulfonyl fluoride (Beyotime Institute of Biotechnology) and incubated for $30 \mathrm{~min}$ on ice. Following centrifugation at $12,000 \mathrm{x} g$ for $15 \mathrm{~min}$, the supernatants were used for protein concentration determination according to a Pierce bicinchoninic acid protein assay kit (Thermo Fisher Scientific, Inc.). Equal amounts of proteins $(20 \mu \mathrm{g})$ were fractionated on SDS-polyacrylamide gels (10\%) and transferred electrophoretically onto polyvinylidene fluoride (EMD Millipore, Billerica, MA, USA) membranes. Membranes were blocked with blocking buffer at room temperature (Beyotime Institute of Biotechnology) and probed overnight with primary mouse anti-cyclin D1 (1:1,000), P21 (1:500), Bax (1:2,000), $\operatorname{Bcl}-2(1: 1,000)$, and $\beta$-actin $(1: 1,000)$ antibodies at $4^{\circ} \mathrm{C}$. Subsequently, membranes were incubated with the HRP-conjugated secondary antibody $(1: 1,000)$ for $1 \mathrm{~h}$ at room temperature, and the signal was detected using a quantitative chemiluminescent WesternBright Quantum kit (Advansta, Inc., Menlo Park, CA, USA).

Statistical analysis. Data analysis was performed with the Student's $t$-test for paired comparison and one-way analysis of variance followed by Tukey's test using GraphPad Prism 4 software (GraphPad Software, Inc., La Jolla, CA, USA). The data are presented as the mean \pm standard deviation and are representative of three individual experiments. $\mathrm{P}<0.05$ was considered to indicate a statistically significant difference.

\section{Results}

Effects of berberine on the growth of MCF-7 and MCF-7/TAM cells. To determine the concentration- and time-dependent effects of berberine exposure, MCF-7 and tamoxifen-resistant MCF-7/TAM cells were treated with increasing doses of berberine for 24,48 and $72 \mathrm{~h}$. 

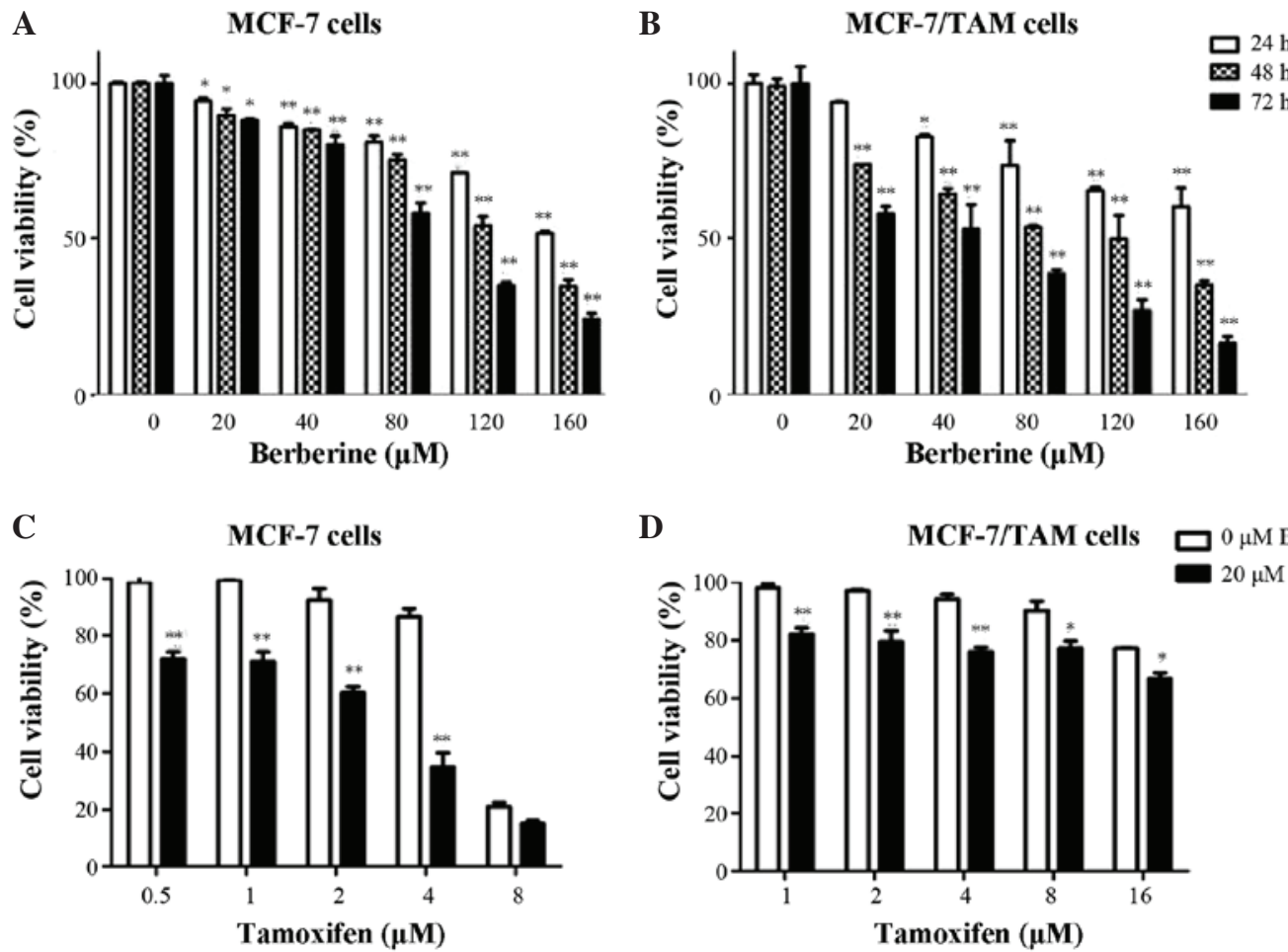

Figure 1. Time- and dose-dependent growth inhibition effect of berberine and tamoxifen. (A) MCF-7 and (B) tamoxifen-resistant MCF-7/TAM cells were treated with increasing doses of berberine for 24,48 and $72 \mathrm{~h}$. Effects of combined treatment of berberine with tamoxifen on the growth of (C) MCF-7 and (D) MCF-7/TAM cells. Cells were treated with various concentrations of tamoxifen with or without berberine for $48 \mathrm{~h}$. Cells viability was then determined by 3-(4,5-dimethylthiazol-2-yl)-5-(3-carboxymethoxyphenyl)-2-(4-sulfophenyl)-2H-tetrazolium assay. ${ }^{*} \mathrm{P}<0.05,{ }^{* * *} \mathrm{P}<0.01$ vs. $0 \mu \mathrm{M}$ berberine group.

Cell proliferation changes were evaluated by MTS assay. As presented in Fig. 1A and B, berberine demonstrated dose- and time-dependent anti-proliferative activity in tamoxifen-sensitive MCF-7 and resistant MCF-7/TAM cells. The $\mathrm{IC}_{50}$ values of berberine for MCF-7 and MCF-7/TAM cells were approximately 130.3 and $99.7 \mu \mathrm{M}$, respectively. MCF-7/TAM cells were more sensitive to berberine compared with MCF-7 cells. Collateral sensitivity is a phenomenon that when a cell population is resistant to certain drugs, it is more sensitive to others. In the subsequent combined studies, $20 \mu \mathrm{M}$ berberine was used, which is also quite selective as it was previously described to be much less cytotoxic against a non-tumorigenic breast cancer cell (w). The results of the present study indicate that berberine exerts potent anti-proliferation activity in MCF-7 breast cancer cells, whether or not they are resistant to tamoxifen.

Co-treatment of berberine and tamoxifen reduces cell viability in MCF-7 and MCF-7/TAM cells. To determine whether combined treatment of tamoxifen and berberine exerts an enhanced anti-cancer effect on breast cancer cells, cell proliferation was determined by MTS assay in MCF-7 and MCF-7/TAM cells treated with tamoxifen alone, or combined with berberine for $48 \mathrm{~h}$. As demonstrated in Fig. 1C and D, the combined treatment of tamoxifen and berberine resulted in synergistic inhibitory effects on MCF-7 and MCF-7/TAM cells. When used alone, tamoxifen $(1 \mu \mathrm{M})$ induced a $0.87 \pm 0.24 \%$ inhibitory effect on MCF-7 cell viability, whereas combined use with $20 \mu \mathrm{M}$ berberine resulted in an inhibitory effect of $29 \pm 3.25 \%(\mathrm{P}<0.01)$. Berberine also significantly enhanced $1 \mu \mathrm{M}$ tamoxifen-induced cell proliferation inhibition from $1.87 \pm 1.24 \%$ to $17.87 \pm 2.05 \%$
$(\mathrm{P}<0.01)$ in MCF-7/TAM cells. These results clearly demonstrated that berberine increased the sensitivity of MCF-7 and MCF-7/TAM cells to tamoxifen compared with tamoxifen alone.

Co-treatment of berberine and tamoxifen induces cell cycle arrest in MCF-7 and MCF-7/TAM cells. To assess whether combined treatment can induce cell cycle arrest in MCF-7 and MCF-7/TAM cells, the cell cycle distribution was examined by flow cytometry analysis. As demonstrated in Fig. 2A, tamoxifen marginally increased the number of MCF-7 cells in the G1 phase. Berberine alone or in combination with tamoxifen significantly increased the number of MCF-7 cells in G1 phase compared with control or tamoxifen alone, respectively $(\mathrm{P}<0.01)$. Similar results were also observed in the MCF-7/TAM cells. As demonstrated in Fig. 2B, $89.07 \pm 1.26 \%$ of the cells treated with berberine and tamoxifen combined were in the G1 phase, which was significantly higher compared with the corresponding percentage of tamoxifen-treated cells $(73.29 \% \pm 1.14 ; \mathrm{P}<0.01)$, berberine-treated cells $(83.93 \pm 2.60 \% ; \mathrm{P}<0.05)$ or vehicle control treated cells $(63.69 \pm 1.61 \% ; \mathrm{P}<0.01)$. The results suggested that co-treatment of tamoxifen and berberine produced an increase in the number of cells in G1 phase compared with tamoxifen alone, clearly demonstrating an effect on G1 cell cycle arrest.

Co-treatment of berberine and tamoxifen induced cell apoptosis in MCF-7 and MCF-7/TAM cells. To determine whether the cell growth inhibition was mediated by apoptosis, cells were stained with Annexin V-FITC/PI following treatment with berberine and tamoxifen. As demonstrated in Fig. 3, each compound treatment produced an observable increase in the percentage of apoptotic cells. It was demonstrated that 
A
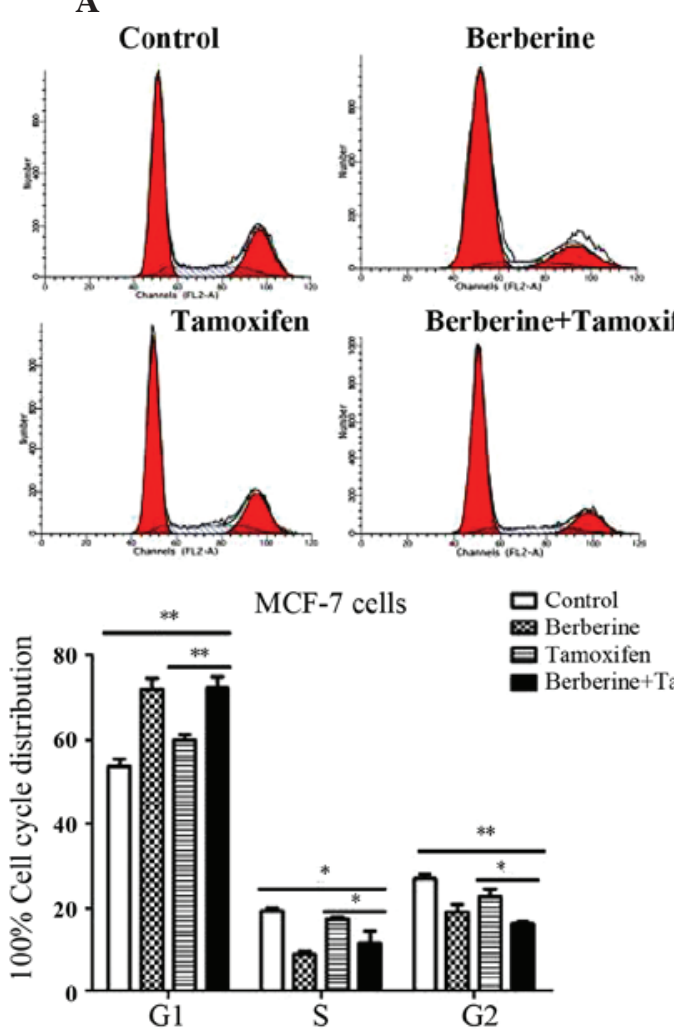

B
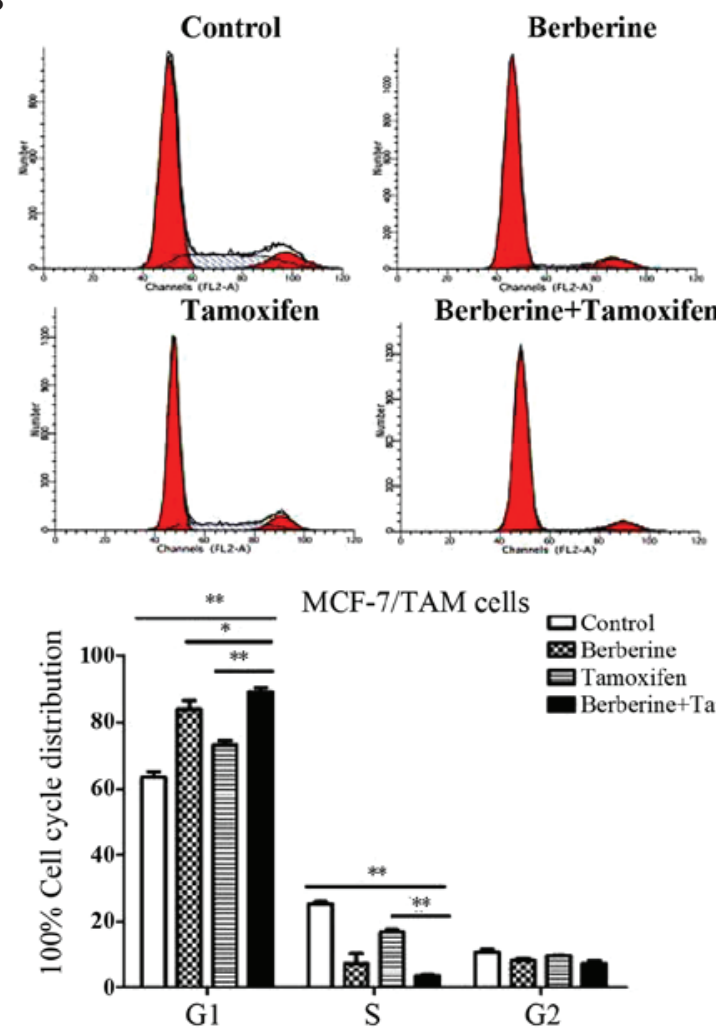

Figure 2. Effects of combined treatment with berberine and tamoxifen on cell cycle distribution. (A) MCF-7 and (B) tamoxifen-resistant MCF-7/TAM cells were treated with $20 \mu \mathrm{M}$ berberine, $1 \mu \mathrm{M}$ tamoxifen alone or their combination for $48 \mathrm{~h}$. The cell cycle of the samples was evaluated by flow cytometry. ${ }^{*} \mathrm{P}<0.05,{ }^{* *} \mathrm{P}<0.01$, comparison indicated by brackets.

,
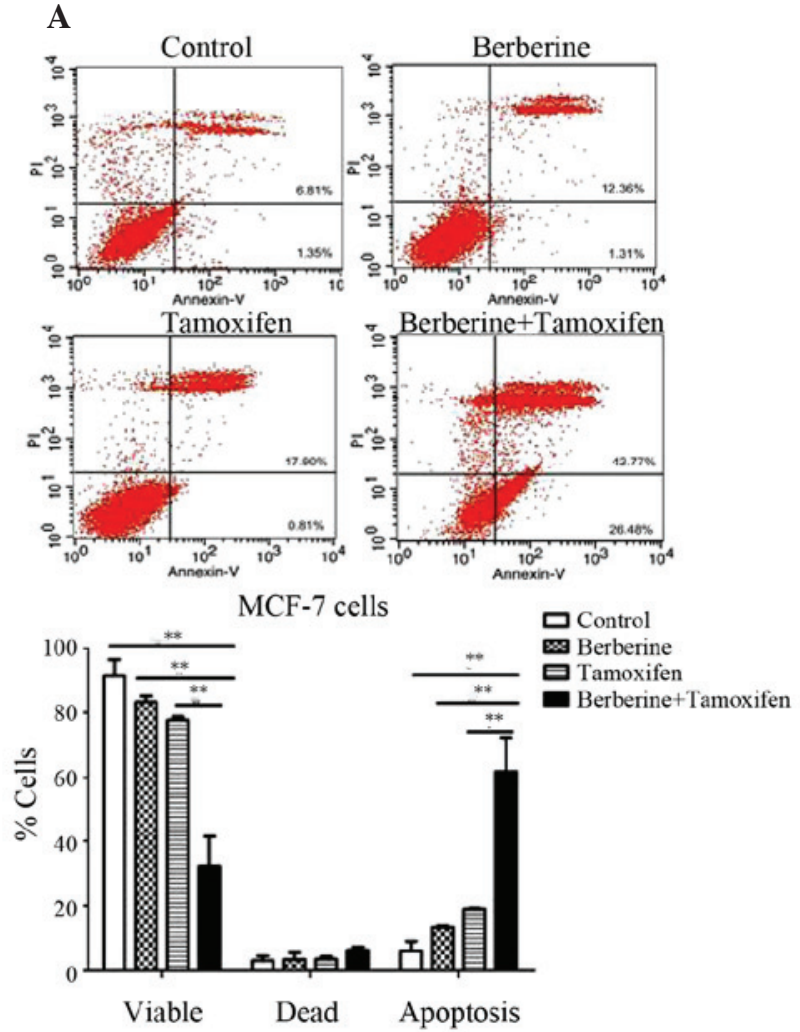

B
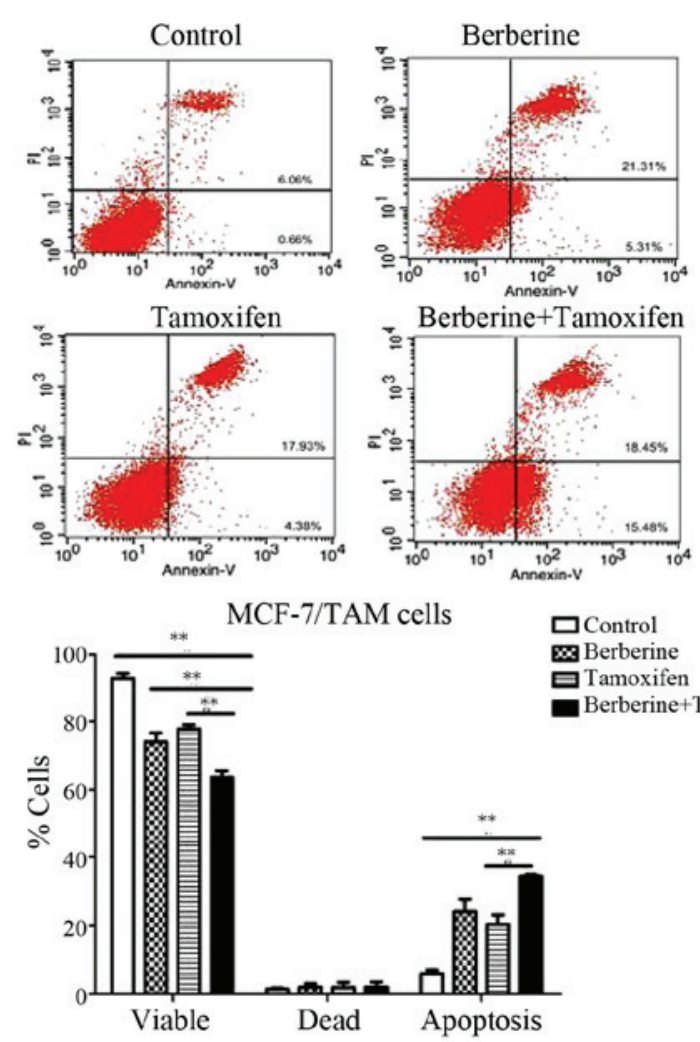

Figure 3. Effects of combined treatment of berberine with tamoxifen on cell apoptosis. (A) MCF-7 and (B) tamoxifen-resistant MCF-7/TAM cells were treated with $20 \mu \mathrm{M}$ berberine, $1 \mu \mathrm{M}$ tamoxifen alone or their combination for $48 \mathrm{~h}$. Apoptosis was evaluated by flow cytometry. ${ }^{* *} \mathrm{P}<0.01$, comparison indicated by brackets. 
$\mathbf{A}$

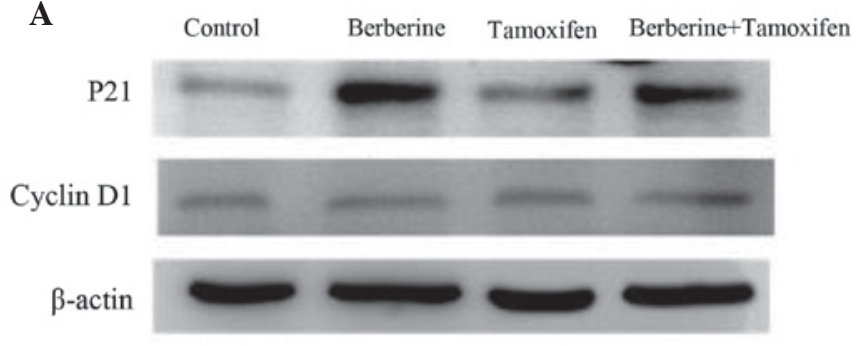

B P21

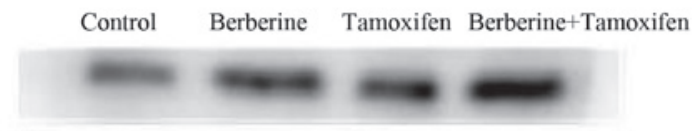

Cyclin D1

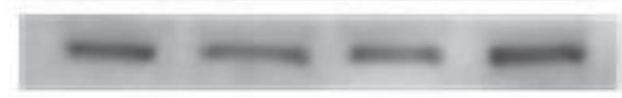

$\beta$-actin

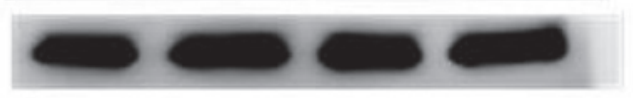

Figure 4. Effects of combined treatment of berberine with tamoxifen on P21 and cyclin D1 protein expression. (A) MCF-7 and (B) tamoxifen-resistant MCF-7/TAM cells were treated with $20 \mu \mathrm{M}$ berberine, $1 \mu \mathrm{M}$ tamoxifen alone or their combination for $48 \mathrm{~h}$, then P21 and cyclin D1 protein expression was detected by western blot analysis.

$20 \mu \mathrm{M}$ berberine and $1 \mu \mathrm{M}$ tamoxifen increased apoptosis to $61.8 \pm 7.47 \%$ compared with $18.9 \pm 0.17 \%$ in tamoxifen only-treated MCF-7 cells $(\mathrm{P}<0.01)$. Furthermore, the number of apoptotic MCF-7/TAM cells was increased in the berberine + tamoxifen group $(20.4 \% \pm 1.95)$ compared with the tamoxifen only group $(34.2 \% \pm 0.32 ; \mathrm{P}<0.01)$ after $48 \mathrm{~h}$ treatment. The results demonstrated that co-treatment is more effective in activating stimulating compared with tamoxifen alone.

Effects of berberine and tamoxifen on $P 21$ and cyclin D1 protein expression. To assess the cell G1 arrest mechanism induced by the combined treatment, western blot analysis of the cell cycle-associated proteins, P21 and cyclin D1, was performed. As demonstrated in Fig. 4, berberine treatment alone efficiently induced the protein expression levels of P21 in MCF-7 and tamoxifen-resistant MCF-7/TAM cells compared with control, whereas tamoxifen alone only marginally increased the levels of P21 in the cell lines. Tamoxifen in combination with berberine markedly increased the tamoxifen-induced level of P21 in MCF-7 and MCF-7/TAM cells. No change was observed in the protein expression level of cyclin D1 in any of the treatment groups. These results suggested that upregulation of P21 may be involved in cell cycle arrest by tamoxifen and berberine in MCF-7 and MCF-7/TAM cells.

Effects of berberine and tamoxifen on Bax and Bcl-2 protein expression. The apoptotic mechanism involved in the cell death response to berberine and tamoxifen was further examined by monitoring the expression levels of anti-apoptotic protein, Bcl-2, and the pro-apoptotic protein, Bax, via western blotting. As demonstrated in Fig. 5, combined treatment efficiently inhibited the protein levels of Bcl-2 in MCF-7 and tamoxifen-resistant MCF-7/TAM cells compared with the control and tamoxifen groups. Notably, a marked increase in Bax
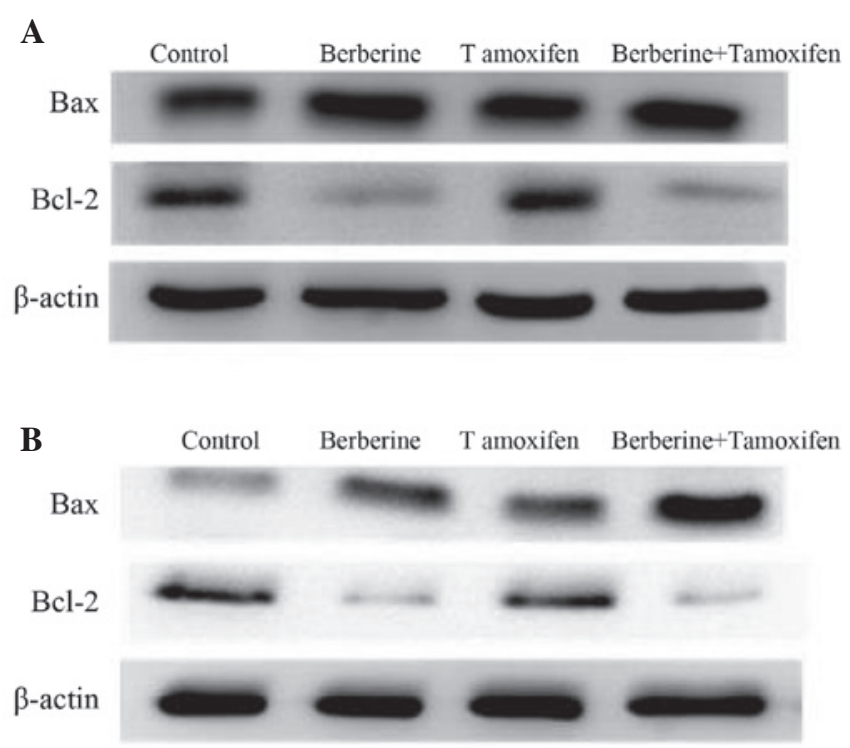

Figure 5. Effects of combined treatment of berberine with tamoxifen on Bax and Bcl-2 protein expression. (A) MCF-7 and (B) tamoxifen-resistant MCF-7/TAM cells were treated with $20 \mu \mathrm{M}$ berberine, $1 \mu \mathrm{M}$ tamoxifen alone or their combination for $48 \mathrm{~h}$, then Bax and Bcl-2 protein expression was detected by western blot analysis. Bax, Bcl-2 associated X protein; Bcl-2, B-cell CLL/lymphoma 2.

expression was observed in the MCF-7/TAM cells combined treatment group, whereas no change in Bax protein expression was observed in MCF-7 cells. Together, these results demonstrated that tamoxifen + berberine-induced apoptosis in breast cancer cells may be mediated by Bax/Bcl-2 upregulation.

\section{Discussion}

Breast cancer remains a worldwide public health concern, and a major cause of morbidity and mortality among females. Endocrine therapies have permitted important progress for the treatment of ER-positive breast cancer. Despite of being a powerful selective ER antagonist, innate or acquired resistance to tamoxifen is a major problem for anti-estrogen therapy $(4,5)$. Thus, it is necessary to develop combined treatments to enhance the efficacy of tamoxifen.

Natural compounds from plants are increasingly being considered as potential anti-cancer agents. Berberine, an isoquinoline alkaloid, has previously been demonstrated to regulate multiple targets and used to enhance targeted therapy sensitization for cancer chemotherapy. For example, doxorubicin and cisplatin, with the combination of berberine, exhibited a higher cytotoxic effect compared with monotherapy in in vivo and in vitro studies $(8,15)$. Berberine has also previously been reported to regulate the expression of cell cycle and apoptosis-associated proteins, including cyclin D1, P21 and Bcl-2. These targets are also understood to be involved in tamoxifen sensitivity and resistance in breast cancer treatment (19-21). It is speculated that berberine may potentially enhance the efficacy of tamoxifen. The results of the current study demonstrated that berberine exerted anti-proliferative effects on MCF-7 and MCF-7/TAM cells, and the co-treatment with berberine and tamoxifen significantly reduced the viability of MCF-7 and MCF-7/TAM cells compared with the tamoxifen applied separately. 
The induction of cell cycle arrest is considered as one of the potential mechanisms of inhibition of cancer development. In order to determine the potential molecular mechanism underlying the synergistic anti-cancer effects of berberine and tamoxifen, the cell cycle distribution was analyzed. The results of the current study indicated that co-treatment significantly induced cell G1 phase arrest in MCF-7 and tamoxifen-resistant MCF-7/TAM cells. The cyclin-dependent kinase inhibitor P21 is involved in the growth and development of breast cancer. It has previously been reported that berberine induced G1 phase arrest by p53-dependent upregulation of P21 (20). P21 has previously been implicated in mediating the sensitivity to tamoxifen in MCF-7 breast cancer cells (22). A previous study demonstrated that loss of P21 function increased tamoxifen resistance (19). The results of the current study demonstrated that berberine + tamoxifen increased the protein expression level of p21 in MCF-7 and MCF-7/TAM cells compared with tamoxifen alone, suggesting that there was functional inactivation of p21 and thus, interference with the effectiveness of tamoxifen.

Apoptosis is crucial in the response of cancer to chemotherapy. The $\mathrm{Bcl}-2 / \mathrm{Bax}$ ratio is important in determining whether a cell will undergo apoptosis or survive (22). The present study demonstrated that the induction of apoptosis in human breast cancer cells by co-treatment was potentially mediated by upregulating the $\mathrm{Bax} / \mathrm{Bcl}-2$ ratio. It was demonstrated that berberine significantly enhanced tamoxifen-induced apoptosis. Bcl-2, an anti-apoptotic protein, has previously been reported to be overexpressed in $28-80 \%$ of patients with breast cancer $(23,24)$. Therefore, the current study determined whether combinational treatment could regulate the ratio of $\mathrm{Bax} / \mathrm{Bcl}-2$. It has been reported that downregulation of Bcl-2 is sufficient to enhance sensitivity to tamoxifen in human breast cancer cells. It has been reported that the downregulation of $\mathrm{Bcl}-2$ is sufficient to enhance sensitivity to tamoxifen in human breast cancer cells (25). These findings suggested that Bax/Bcl-2 upregulation may function as a point of convergence for inducing cell apoptosis. The present study demonstrated that the induction of apoptosis in human breast cancer cells by berberine and tamoxifen co-treatment was potentially mediated by upregulating the $\mathrm{Bax} / \mathrm{Bcl}-2$ ratio.

In the present study, the combined effects of berberine and tamoxifen on growth inhibition were evident. This finding is based on the fact that co-treatment with berberine and tamoxifen significantly reduced the viability of breast cancer cells compared to the tamoxifen treatment alone, Additionally, co-treatment induced cell cycle arrest and increased apoptotic cell death in the current study. The tamoxifen-resistant MCF-7/TAM breast cancer cells exhibited similar responses to tamoxifen-sensitive MCF-7 cells, indicating that berberine in combination with tamoxifen enhanced the growth inhibition of breast cancer cells independent of tamoxifen resistance. These results demonstrated that berberine may be a powerful adjuvant for anti-cancer therapy, particularly when combined with tamoxifen for ER-positive breast cancer treatment. The findings of the present study provide novel evidence for the importance and the effectiveness of combinational drug treatment in anti-cancer chemotherapy. However, in vivo study and further mechanistic analyses are necessary to confirm the effects of this combination.

\section{Acknowledgements}

This research was supported by the National Scientific Foundation of China (grant nos. 81473284 and 81102511), the Natural Science Foundation of Chongqing (grant no. cstc2011jjA10004), and the Funds for Outstanding Young Scholars in Chongqing Medical University (grant nos. CYYQ201301 and CYYQ201401).

\section{References}

1. Torre LA, Bray F, Siegel RL, Ferlay J, Lortet-Tieulent J and Jemal A: Global cancer statistics, 2012. CA Cancer J Clin 65: 87-108, 2015.

2. Cuzick J, Powles T, Veronesi U, Forbes J, Edwards R, Ashley S and Boyle P: Overview of the main outcomes in breast-cancer prevention trials. Lancet 361: 296-300, 2003.

3. Early Breast Cancer Trialists' Collaborative Group (EBCTCG): Effects of chemotherapy and hormonal therapy for early breast cancer on recurrence and 15-year survival: An overview of the randomised trials. Lancet 365: 1687-1717, 2005.

4. Clarke R, Liu MC, Bouker KB, Gu Z, Lee RY, Zhu Y, Skaar TC, Gomez B, O'Brien K, Wang Y, et al: Antiestrogen resistance in breast cancer and the role of estrogen receptor signaling. Oncogene 22: 7316-7339, 2003.

5. Lewis JS and Jordan VC: Selective estrogen receptor modulators (SERMs): Mechanisms of anticarcinogenesis and drug resistance. Mutat Res 591: 247-263, 2005.

6. Jiang M, Huang O, Zhang X, Xie Z, Shen A, Liu H, Geng M and Shen K: Curcumin induces cell death and restores tamoxifen sensitivity in the antiestrogen-resistant breast cancer cell lines MCF-7/LCC2 and MCF-7/LCC9. Molecules 18: 701-720, 2013.

7. Charalambous C, Pitta CA and Constantinou AI: Equol enhances tamoxifen's anti-tumor activity by induction of caspase-mediated apoptosis in MCF-7 breast cancer cells. BMC Cancer 13: 238, 2013.

8. Mittal A, Tabasum S and Singh RP: Berberine in combination with doxorubicin suppresses growth of murine melanoma B16F10 cells in culture and xenograft. Phytomedicine 21: 340-347, 2014.

9. Zhao Q, Wang J, Zou MJ, Hu R, Zhao L, Qiang L, Rong JJ, You QD and Guo QL: Wogonin potentiates the antitumor effects of low dose 5-fluorouracil against gastric cancer through induction of apoptosis by down-regulation of NF-kappaB and regulation of its metabolism. Toxicol Lett 197: 201-210, 2010.

10. Notarbartolo M, Poma P, Perri D, Dusonchet L, Cervello M and D'Alessandro N: Antitumor effects of curcumin, alone or in combination with cisplatin or doxorubicin, on human hepatic cancer cells. Analysis of their possible relationship to changes in NF-kB activation levels and in IAP gene expression. Cancer Lett 224: 53-65, 2005.

11. Wen CJ, Wu LX, Fu LJ, Yu J, Zhang YW, Zhang X and Zhou HH: Genomic screening for targets regulated by berberine in breast cancer cells. Asian Pac J Cancer Prev 14: 6089-6094, 2013.

12. Wang Y, Liu Q, Liu Z, Li B, Sun Z, Zhou H, Zhang X, Gong Y and Shao C: Berberine, a genotoxic alkaloid, induces ATM-Chk1 mediated G2 arrest in prostate cancer cells. Mutat Res 734: 20-29, 2012.

13. Lu B, Hu M, Liu K and Peng J: Cytotoxicity of berberine on human cervical carcinoma HeLa cells through mitochondria, death receptor and MAPK pathways, and in-silico drug-target prediction. Toxicol In Vitro 24: 1482-1490, 2010.

14. Lu B, Zhao J, Xu L, Xu Y, Wang X and Peng J: Identification of molecular target proteins in berberine-treated cervix adenocarcinoma HeLa cells by proteomic and bioinformatic analyses. Phytother Res 26: 646-656, 2012.

15. Youn MJ, So HS, Cho HJ, Kim Y, Lee JH, Sohn JS, Kim YK, Chung SY and Park R: Berberine, a natural product, combined with cisplatin enhanced apoptosis through a mitochondria/caspase-mediated pathway in HeLa cells. Biol Pharm Bull 31: 789-795, 2008.

16. Lin TH, Kuo HC, Chou FP and Lu FJ: Berberine enhances inhibition of glioma tumor cell migration and invasiveness mediated by arsenic trioxide. BMC Cancer 8: 58, 2008. 
17. Fan LX, Liu CM, Gao AH, Zhou YB and Li J: Berberine combined with 2 -deoxy-d-glucose synergistically enhances cancer cell proliferation inhibition via energy depletion and unfolded protein response disruption. Biochim Biophys Acta 1830: 5175-5183, 2013.

18. Patil JB,Kim J and Jayaprakasha GK: Berberine induces apoptosis in breast cancer cells (MCF-7) through mitochondrial-dependent pathway. Eur J Pharmacol 645: 70-78, 2010.

19. Abukhdeir AM, Vitolo MI, Argani P, De Marzo AM, Karakas B, Konishi H, Gustin JP, Lauring J, Garay JP, Pendleton C, et al: Tamoxifen-stimulated growth of breast cancer due to p21 loss. Proc Natl Acad Sci USA 105: 288-293, 2008.

20. Liu Z, Liu Q, Xu B, Wu J, Guo C, Zhu F, Yang Q, Gao G, Gong Y and Shao C: Berberine induces p53-dependent cell cycle arrest and apoptosis of human osteosarcoma cells by inflicting DNA damage. Mutat Res 662: 75-83, 2009.

21. Larsen MS, Bjerre K, Giobbie-Hurder A, Lænkholm AV, Henriksen KL, Ejlertsen B, Lykkesfeldt AE and Rasmussen BB: Prognostic value of $\mathrm{Bcl}-2$ in two independent populations of estrogen receptor positive breast cancer patients treated with adjuvant endocrine therapy. Acta Oncol 51: 781-789, 2012.
22. Lv L, Zheng L, Dong D, Xu L, Yin L, Xu Y, Qi Y, Han X and Peng J: Dioscin, a natural steroid saponin, induces apoptosis and DNA damage through reactive oxygen species: A potential new drug for treatment of glioblastoma multiforme. Food Chem Toxicol 59: 657-669, 2013.

23. Callagy GM, Pharoah PD, Pinder SE, Hsu FD, Nielsen TO, Ragaz J, Ellis IO, Huntsman D and Caldas C: Bcl-2 is a prognostic marker in breast cancer independently of the Nottingham prognostic index. Clin Cancer Res 12: 2468-2475, 2006.

24. Cardoso F, Paesmans M, Larsimont D, Durbecq V, Bernard-Marty C, Rouas G, Dolci S, Sotiriou C, Piccart MJ and Di Leo A: Potential predictive value of $\mathrm{Bcl}-2$ for response to tamoxifen in the adjuvant setting of node-positive breast cancer. Clin Breast Cancer 5: 364-369, 2004.

25. Xu C, Kong X, Wang H, Zhang N, Kong X, Ding X, Li X and Yang Q: MTDH mediates estrogen-independent growth and tamoxifen resistance by down-regulating PTEN in MCF-7 breast cancer cells. Cell Physiol Biochem 33: 1557-1567, 2014. 